\title{
Prognostic impact of myocardial contraction fraction in patients undergoing transcatheter aortic valve replacement for aortic stenosis
}

\author{
Francisco J. Romeo ${ }^{1}$, Ignacio M. Seropian ${ }^{1}$, Sameer Arora ${ }^{2}, J^{\prime}$. P. Vavalle ${ }^{2}$, Mariano Falconi ${ }^{3}$, Pablo Oberti ${ }^{3}$, \\ Vadim Kotowicz ${ }^{4}$, Carla R. Agatiello ${ }^{1}$, Daniel H. Berrocal ${ }^{1}$ \\ ${ }^{1}$ Division of Interventional Cardiology, Hospital Italiano de Buenos Aires, Buenos Aires, Argentina; ${ }^{2}$ Division of Cardiology, University of North \\ Carolina, Chapel Hill, NC, USA; ${ }^{3}$ Division of Cardiology, ${ }^{4}$ Division of Cardiovascular Surgery, Hospital Italiano de Buenos Aires, Buenos Aires, \\ Argentina \\ Contributions: (I) Conception and design: FJ Romeo; (II) Administrative support: FJ Romeo; (III) Provision of study material or patients: All \\ authors; (IV) Collection and assembly of data: FJ Romeo, IM Seropian; (V) Data analysis and interpretation: FJ Romeo, IM Seropian, S Arora; (VI) \\ Manuscript writing: All authors; (VII) Final approval of manuscript: All authors. \\ Correspondence to: Francisco J. Romeo, MD. Department of Interventional Cardiology, Hospital Italiano de Buenos Aires, Buenos Aires C1199, \\ Argentina. Email: francisco.romeo@hospitalitaliano.org.ar.
}

Background: Myocardial contraction fraction (MCF), a volumetric measurement of myocardial shortening, may help to improve risk stratification in patients with severe aortic stenosis (AS) referred for transcatheter aortic valve replacement (TAVR) especially in those with preserved left ventricular ejection fraction (LVEF). We investigated the association between MCF and 1-year all-cause mortality in patients with severe AS who underwent TAVR.

Methods: MCF was calculated as the ratio of stroke volume (SV) to myocardial volume. Patients referred for TAVR from 2011 to 2015 were eligible for inclusion and were divided into two groups according to the estimated MCF (MCF $\leq 30 \%$ vs. MCF >30\%). The primary endpoint was 1-year all-cause mortality. A Cox regression analysis was performed for independent risk factors of mortality. Receiver operating curve (ROC) was performed for assessing the best cut-off point of MCF for predicting the primary outcome [area under the curve (AUC) 0.60; 95\% confidence interval (CI): 0.453-0.725]. Baseline patient and echo characteristics were included for multivariate analysis.

Results: Of 126 patients (mean age $82 \pm 5$ years, $45.2 \%$ male), $44.4 \%$ showed MCF $\leq 30 \%$. Patient with reduced MCF showed higher body mass index $\left(28.1 \pm 5.8\right.$ vs. $\left.26.0 \pm 4.5 \mathrm{~kg} / \mathrm{m}^{2}, \mathrm{P}=0.031\right)$, higher surgical EuroScore II (6.2 \pm 4.5 vs. $4.7 \pm 3.2, \mathrm{P}=0.032)$, lower LVEF $(54.2 \% \pm 11.9 \%$ vs. $58.5 \% \pm 10.8 \%, \mathrm{P}=0.042)$ and more severe AS (indexed aortic valve area $0.40 \pm 0.09$ vs. $0.45 \pm 0.10 \mathrm{~cm}^{2} / \mathrm{m}^{2}, \mathrm{P}=0.030$ ). The median followup was of 14 [3.5-33] months, and $16 \%$ of patients died. Patients with MCF $\leq 30 \%$ showed significantly increased all-cause mortality (Log-rank $\mathrm{P}=0.002$ ). In a multivariate model adjusting for clinical and echo variables, $\mathrm{MCF} \leq 30 \%$ was independently associated with increased risk for all-cause 1-year mortality [hazard ratio (HR) $2.76,95 \% \mathrm{CI}: 1.03-7.77, \mathrm{P}=0.04]$.

Conclusions: In a population of patients undergoing TAVR, MCF $\leq 30 \%$ was independently associated with increased mortality.

Keywords: Myocardial contraction fraction (MCF); transcatheter aortic valve replacement (TAVR); aortic stenosis (AS)

Submitted Apr 06, 2019. Accepted for publication May 20, 2019.

doi: $10.21037 / \mathrm{cdt} .2019 .05 .02$

View this article at: http://dx.doi.org/10.21037/cdt.2019.05.02 


\section{Introduction}

Transcatheter aortic valve replacement (TAVR) as a therapeutic strategy for patients with severe symptomatic aortic stenosis (AS) has become the preferred alternative to surgical valve replacement (SAVR) for inoperable and highrisk patients. For this clinical scenario, TAVR has been shown to provide substantial benefit in both survival and quality of life when compared with medical therapy alone and similar intermediate-term survival when compared with SAVR (1-4).

Left ventricular ejection fraction (LVEF) has remained as the gold standard marker for LV systolic function in different cardiac diseases. In acute myocardial infarction (AMI) (5) and dilated cardiomyopathy (DCM) (6), LVEF remains as the strongest predictor for patient survival at follow-up. Moreover, in severe AS, an LVEF decrease is an indication for valve replacement/TAVR (7).

Left ventricular (LV) systolic dysfunction in patients suitable for TAVR ranges between $6-11 \%$ and $27-46 \%$ when defined using LVEF $\leq 30 \%$ or LVEF between $30 \%$ and $50 \%$ respectively (8). However, LVEF may be inaccurate for predicting outcomes in patients with symptomatic severe AS. A subsequent analysis from the PARTNER trial was not able to find low baseline LVEF as an independent risk factor on both post-TAVR and SAVR outcomes (9). Due to the limitations of LVEF, and in order to predict outcomes in patients with AS undergoing TAVR, research groups have reinforced to look for new echocardiographic parameters for indexing LV systolic function to geometric patterns, improving accuracy.

Myocardial contraction fraction (MCF) is defined as the ratio of stroke volume (SV) to myocardial volume. $\mathrm{LV}$ myocardial volume is defined as $\mathrm{LV}$ myocardial mass divided by the mean density of myocardium, i.e., $1.05 \mathrm{~g} / \mathrm{mL}$.

$$
M C F=\frac{L V \text { Stroke volume }(m L)}{L V \text { Myocardial volume }(m L)} * 100[\%]
$$

To express the fraction as an index, the result was multiplied by 100 . Indeed, this equation can be regarded as a volumetric measurement of myocardial shortening independent from LV dimensions, wall thicknesses and geometric patterns, discriminating adaptive from maladaptive hypertrophic response to chronic pressure overload (10). Furthermore, MCF shows a strong correlation with global longitudinal strain (GLS) (11), and, recently, MCF has outperformed LVEF in patients with cardiac amyloidosis (CA) (12) and has shown prognostic value for predicting incident heart failure (HF) (13), and cardiac events in patients with non-ischaemic DCM (14).
However, the role of MCF in patients undergoing TAVR is unknown.

In the present study, we aim to describe the prognostic value of MCF in patients undergoing TAVR for severe symptomatic AS.

\section{Methods}

\section{Study design and data collection}

We retrospectively analyzed both baseline clinical characteristics and echocardiographic imaging data collected in 141 consecutive patients who underwent TAVR with a balloon-expandable or self-expandable prosthetic valve for symptomatic severe AS between January 2011 and December 2015 at the Hospital Italiano in Buenos Aires, Argentina.

This study was approved by the Hospital Italiano Institutional Review Board (IRB No. 00010193), and the requirement for informed consent was waived. The following were exclusion criteria: "valve in-valve" procedure, severe aortic regurgitation, recent myocardial infarction, congenital unicuspid or bicuspid valve, incomplete preprocedural Doppler echocardiographic data, including patients with only transesophageal echocardiograms (TEE) at baseline.

Electronic medical records were used for collecting both patients' baseline characteristics as well as operative data including hospital stays. The index echocardiographic examination was defined as the last transthoracic echocardiographic (TTE) study before TAVR. Paravalvular aortic insufficiency after TAVR was evaluated according to the Valve Academic Research Consortium-2 criteria on echocardiograms performed 30 days post-TAVR. When 30-day echocardiograms were not available, discharge echocardiograms were used.

All patients were followed until death (all-cause mortality) or last contact within the first year after TAVR, at which time they were censored.

\section{Echocardiography imaging}

The whole cohort underwent an exhaustive TTE exam with IE33 or HD15 (Philips Healthcare, Amsterdam, The Netherlands). All TTE exams were performed and analyzed in the same lab by the same team of cardiologists following the recommendations of the American Society of Echocardiography (ASE) and the European Association of Cardiovascular Imaging (EACVI) (15). The TTE obtained 6 to 12 months after TAVR was used to assess late changes 
in MCF.

Bland-Altman plots were not drawn for the interobserver agreement during echo data collection, however, echo data were analyzed by two experienced cardiac ultrasonographers with experience in the field using the same laboratory and equipment.

The standard measurement of cardiac size and function were performed per ASE/EACVI recommendations (16).

AS severity was defined according to peak velocity, mean gradient and calculation of the aortic valve area (AVA) using the continuity equation: AVA $=\mathrm{LV}$ outflow tract $(\mathrm{LVOT})$ area $x$ ratio of LVOT to aortic valve time-velocity integral, where LVOT area is the cross-sectional area of the LVOT. AVA was indexed to the patient' s body surface area. All patients had an indexed AVA of $<0.6 \mathrm{~cm}^{2} / \mathrm{m}^{2}$. SV was calculated by pulsed wave Doppler using the following formula: LVOT area $\times$ LVOT time integral velocity $(\mathrm{VTI})=\pi(\text { diameter } / 2)^{2}$ $\times$ LVOT VTI. In order to avoid errors in measurements, the LVOT diameter and velocity used to calculate SV were measured just below the insertion of aortic valve cusps before the implantation, whereas after TAVR they were measured just underneath the apical border of the stent.

LV end-systolic (ESV) and end-diastolic (EDV) volumes were indirectly measured through calculations based on LV dimensions measured according to ASE guidelines and previously validated techniques with the use of linear dimensions from two dimensional echocardiography. LV EDV was estimated as $4.5 £$ $\sqrt{[\mathrm{LV} \text { end-diastolic dimension (LVEDD) }]}$ and LV ESV was estimated as $3.72 £$ (LV end-systolic dimension).

Manual tracing of the LV cavity endocardium at endsystole and end-diastole, from both the apical 4-chamber view and the apical 2-chamber view were obtained in order to estimate LVEF (biplane Simpson method), except when poor acoustic window precluded reliable delineation of endocardial borders. In this situation, a visual estimation of LVEF was performed. LV mass was calculated with the linear method, using the corrected formula of the ASE - European Association of Echocardiography $(\mathrm{EAE}) / \mathrm{EACVI}$ as follows: left ventricular mass $(\mathrm{LVM})=$ $0.8^{*} 1.04^{*}$ \{[interventricular septal wall thickness (IVST) + left ventricular internal diameter (LVID) + posterior wall thickness $\left.(\mathrm{PWT})]^{3}-\mathrm{LVID}^{3}\right\}+0.6$. LVM was further indexed to BSA [LVM index (LVMi)] or to a 2.7 power of height $\left(\mathrm{LVMi}^{2.7}\right)$, as previously described to adjust for the effect of obesity on BSA. LVH was defined as LVMi $>115 \mathrm{~g} / \mathrm{m}^{2}$ in men and $>95 \mathrm{~g} / \mathrm{m}^{2}$ in women.

Myocardial contraction fraction was calculated as the ratio of SV to myocardial volume ( $\mathrm{LV}$ mass/mean density of myocardium), which were calculated from linear dimensions in the parasternal long-axis view. LV mass determination by different imaging modalities is based on the $\mathrm{LV}$ shell volume (LVShV), which is the difference between the epicardial and endocardial volumes. The $\mathrm{LVShV}$ is subsequently converted to mass by multiplying it by the density of myocardial tissue. The clinically accepted value of myocardial tissue density is $1.055 \mathrm{~g} / \mathrm{mL}$.

\section{Statistical analysis}

Continuous variables were presented as mean $\pm \mathrm{SD}$ or median and categorical variables were summarized as counts (frequency percentages). MCF groups were compared with one-way ANOVA followed by a Tukey's post-hoc test when appropriate. Categorical data were compared with the $\mathrm{Chi}^{2}$ test or Fisher's exact test when appropriate. Baseline and follow-up comparison of echocardiographic parameters of the same patients was performed through paired Student's $t$-test. Survival curves were presented as Kaplan-Meier curves, and the log-rank test was used for comparison between groups. The date of TAVR was considered the start date.

All of the analyses were considered significant at a twotailed $\mathrm{P}$ value of $\leq 0.05$.

The effect of the clinical and Doppler echocardiographic variables on survival was assessed with Cox proportional hazards regression models for cumulative all-cause 1-year mortality. All non-collinear variables with a $\mathrm{P}$ value $\leq 0.1$ on univariable analysis were included in the multivariable model, and several model selection methods were employed (backward, forward, and stepwise). For constructing a multivariate model, no more than four variables were analyzed in order to avoid overfitting.

Using MCF as a diagnostic marker for predicting 1 -year all-cause mortality, we estimated the area under the corresponding receiver operating curve (ROC) curve (C-statistic) with corresponding $95 \%$ confidence interval (CI).

All statistical tests were performed using statistical software SPSS 23.0 for Microsoft (SPSS Inc.; IBM, Chicago, IL, USA) and GraphPad Prism version 7.0 for Microsoft (GraphPad Software, La Jolla, CA, USA).

\section{Results}

From a total of 141 patients, 126 patients were included. 
Table 1 Baseline characteristics in patients with severe AS with low and high MCF

\begin{tabular}{|c|c|c|c|c|}
\hline Characteristics & All $(n=126)$ & $M C F \leq 30 \%(n=56)$ & MCF >30\% $(n=70)$ & $P$ value \\
\hline Age, mean $\pm S D$, years & $82.6 \pm 5.4$ & $82.9 \pm 5.1$ & $82.4 \pm 5.8$ & 0.656 \\
\hline Male sex, n (\%) & $56(44.4)$ & $20(35.7)$ & $36(51.4)$ & 0.124 \\
\hline $\mathrm{BMI}$, mean $\pm \mathrm{SD}, \mathrm{kg} / \mathrm{m}^{2}$ & $27.0 \pm 5.2$ & $28.1 \pm 5.8$ & $26.0 \pm 4.5$ & 0.031 \\
\hline 1 & $5(4.0)$ & $4(7.1)$ & $1(1.4)$ & - \\
\hline II & $81(64.3)$ & $36(64.3)$ & $45(64.3)$ & 0.252 \\
\hline III & $35(27.8)$ & $13(23.2)$ & $22(31.4)$ & - \\
\hline IV & $5(4.0)$ & $3(5.4)$ & $2(2.9)$ & - \\
\hline D2: Low-flow, low-gradient AS with reduced LVEF & $15(11.9)$ & $9(16.1)$ & $6(8.6)$ & 0.355 \\
\hline D3: Low-flow, low-gradient AS with normal LVEF & $29(23.0)$ & $11(19.6)$ & $18(25.7)$ & - \\
\hline \multicolumn{5}{|l|}{ Clinical parameters } \\
\hline Hypertension, n (\%) & $112(88.9)$ & $50(89.3)$ & $62(88.6)$ & 0.511 \\
\hline Diabetes mellitus, $\mathrm{n}(\%)$ & $28(22.2)$ & $13(23.2)$ & $15(21.4)$ & 0.716 \\
\hline Chronic lung disease, $\mathrm{n}(\%)$ & $11(8.7)$ & $4(7.1)$ & $7(10.0)$ & 0.619 \\
\hline Coronary artery disease, $\mathrm{n}(\%)$ & $68(54.0)$ & $30(53.6)$ & $38(54.3)$ & 0.866 \\
\hline Peripheral vascular disease, $\mathrm{n}(\%)$ & $30(23.8)$ & $13(23.2)$ & $17(24.3)$ & 0.989 \\
\hline Estimated GFR, median [range], $\mathrm{mL} / \mathrm{min}$ & $67.2[49.0-82.9]$ & $70.8[54.5-82.4]$ & $66.6[38.5-85.0]$ & 0.133 \\
\hline
\end{tabular}

BMI, body mass index, GFR, glomerular filtration rate; MCF, myocardial contraction fraction; AS, aortic stenosis; SD, standard deviation; LVEF, left ventricular ejection fraction; NT-pro-BNP, N-terminal pro-brain natriuretic peptide.

They were elderly (mean age $82 \pm 5$ years), $45.2 \%$ male $(\mathrm{N}=57)$ with severe cardiac symptoms $(70 \%$ had New York Heart Association functional class $>2$ ) and frequent comorbid conditions. On average, $\mathrm{N}$-terminal pro-brain natriuretic peptide (NT-pro-BNP) was elevated with a median of $1,002[451-2,441] \mathrm{pg} / \mathrm{mL}$, and a mean LVEF of $56 \% \pm 11 \%$.

ROC curve was performed for assessing the optimal cutpoint and its corresponding Youden Index [area under the curve (AUC) $0.60 ; 95 \%$ CI: 0.453-0.725]. Patients with severe AS referred for TAVR were divided into two groups according to the estimated MCF cutoff (MCF $\leq 30 \% v s$. MCF $>30 \%)$. Fifty-six (44.4\%) patients had MCF $\leq 30 \%$. Patients with preoperative MCF $\leq 30 \%$ showed higher body mass index and higher surgical EuroScore II. Table 1 shows the comparison of the patients' baseline characteristics according to MCF cutoff value.

Baseline echocardiographic characteristics are listed in Table 2. Patients with low MCF showed lower LVEF $(54.2 \% \pm 11.9 \%$ vs. $58.5 \% \pm 10.8 \%, \mathrm{P}=0.042)$ and more severe 
Table 2 Baseline echocardiographic data in patients with severe AS with low and high MCF

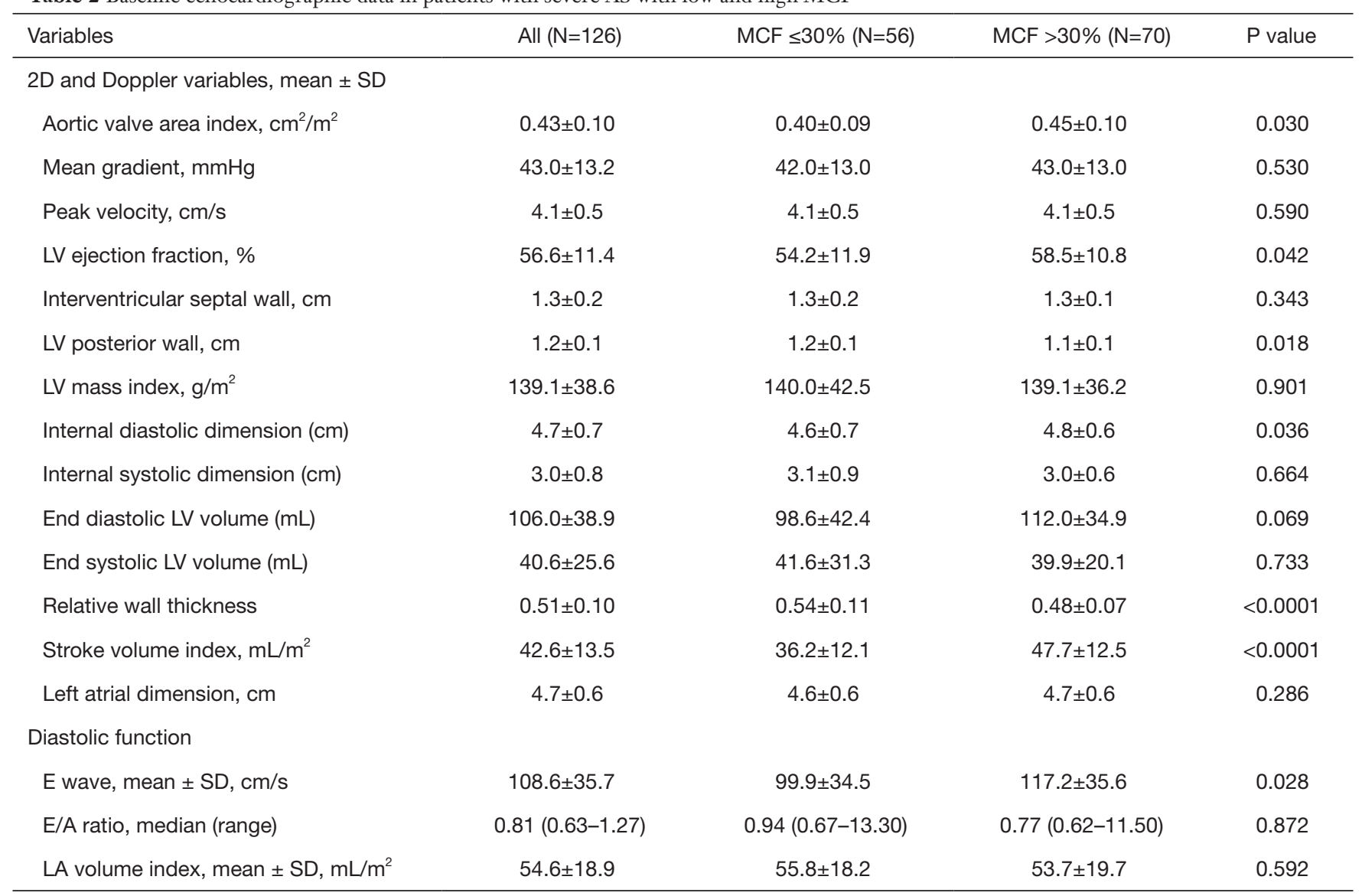

MCF, myocardial contraction fraction; SD, standard deviation; LV, left ventricular; LA, left atrial.

AS (AVA index $0.40 \pm 0.09$ vs. $0.45 \pm 0.10 \mathrm{~cm}^{2} / \mathrm{m}^{2}, \mathrm{P}=0.030$ ). Moreover, these patients presented thicker $\mathrm{LV}$ posterior walls, lower $\mathrm{LV}$ internal diastolic dimensions, higher relative wall thickness (RWT), and lower E-wave. Finally, when assessing SV index (SVi), these patients had lower flow (LF) in comparison with the group with MCF $>30 \%(36.2 \pm 12.1$ vs. $\left.47.7 \pm 12.5 \mathrm{~mL} / \mathrm{m}^{2}, \mathrm{P}<0.0001\right)$.

Overall 30-day and 1-year outcomes post-TAVR in both groups are listed in Table 3.

\section{Impact of baseline myocardial contraction fraction on late mortality}

Twenty deaths (15.9\%) occurred within the first year after TAVR. Patients with MCF $\leq 30 \%$ had increased 1 -year mortality compared with those with MCF $>30 \%$ (Figure 1).

The univariable and multivariable analysis is described in Table 4. Both MCF $\leq 30 \%$ and left atrial volume index (LAVI) $\geq 40 \mathrm{~mL} / \mathrm{m}^{2}$ were associated with increased risk of late mortality. In a multivariate model adjusting for age, Euroscore $\geq 5, \mathrm{MCF} \leq 30 \%$ and LAVI $\geq 40 \mathrm{~mL} / \mathrm{m}^{2}$, low MCF was the only independent predictor associated with increased risk for all-cause 1-year mortality (HR 2.76, 95\% CI: $1.03-7.77, \mathrm{P}=0.04)$.

\section{Changes in MCF at follow-up}

Complete baseline and late procedural (LP) Doppler echocardiograms were available in 103 patients $(81.7 \%)$. MCF increased significantly from baseline to late follow-up only in the group with baseline-MCF $\leq 30 \%(22.6 \% \pm 6.4 \%$ to $42.7 \% \pm 17.9 \%, \mathrm{P}<0.0001)$. No significant difference was observed between baseline-MCF and LP-MCF in the group with baseline-MCF $>30 \%(41.8 \% \pm 10.8 \%$ to $39.3 \% \pm 16.5 \%$, $\mathrm{P}=\mathrm{NS}$ ) (Figure 2).

Figure 3 illustrates the evolution of MCF according to the type of approach used for TAVR, that is, transfemoral $(\mathrm{TF}, \mathrm{n}=62,60.2 \%) v s$. transapical (TA) $(\mathrm{n}=41,39.8 \%)$. 
Table 3 Thirty-day and 1-year outcomes according to low and high MCF

\begin{tabular}{|c|c|c|c|c|}
\hline Outcomes data & All $(\mathrm{N}=126)$ & $\mathrm{MCF} \leq 30 \%(\mathrm{~N}=56)$ & MCF $>30 \%(N=70)$ & $P$ value \\
\hline 30-day deaths, n (\%) & $10(7.9)$ & 7 (12.5) & $3(4.3)$ & 0.081 \\
\hline Length of stay (days since TAVR), median [range] & $6[4-8]$ & $6[4-8.5]$ & $6[4-7]$ & 0.185 \\
\hline Stroke, n (\%) & $3(2.4)$ & $2(3.6)$ & $1(1.4)$ & 0.411 \\
\hline Cumulative all-cause mortality within 1 year, $\mathrm{n}(\%)$ & $20(15.9)$ & $15(26.8)$ & $5(7.1)$ & 0.002 \\
\hline Heart failure readmissions within 1 year, $\mathrm{n}(\%)$ & $15(11.9)$ & $6(10.7)$ & 9 (12.9) & 0.784 \\
\hline
\end{tabular}

MCF, myocardial contraction fraction; TAVR, transcatheter aortic valve replacement.

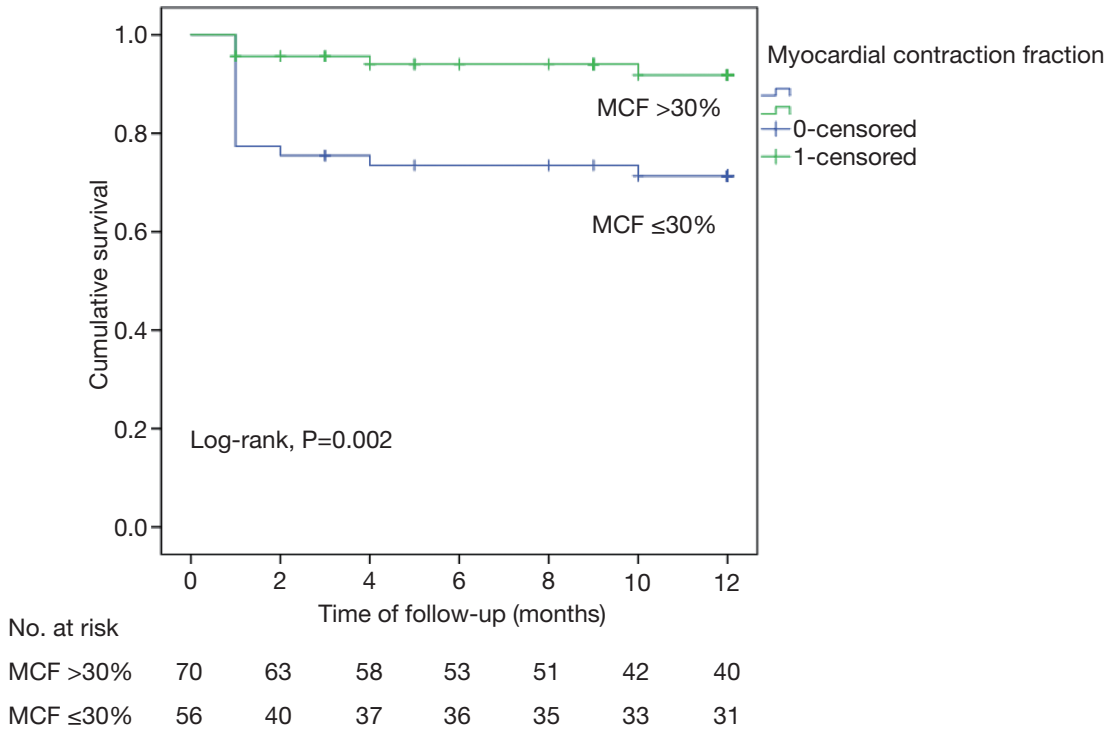

Figure 1 One-year survival based on baseline MCF cutoff. MCF, myocardial contraction fraction.

At baseline and LP, MCF only increased significantly when the TF approach was performed $(34.5 \% \pm 13.8 \%$ to $40.7 \% \pm 14.6 \%, \mathrm{P}=0.03)$. Although patients undergoing a TA approach showed a slight increase in MCF between baseline and LP, this improvement was not statistically significant (35.6 \pm 11.7 to $39.4 \pm 15.2, \mathrm{P}=\mathrm{NS}$ ). As depicted in Figure 4, the evolution of MCF was similar in patients with classical LF (i.e., $\mathrm{LVEF}<50 \%$ and $\mathrm{SVi}<35 \mathrm{~mL} / \mathrm{m}^{2}$ ) and in patients with paradoxical $\mathrm{LF}$ (i.e., $\mathrm{LVEF} \geq 50 \%$ and $\mathrm{SVi}<35 \mathrm{~mL} / \mathrm{m}^{2}$ ).

LVEF was higher in the MCF $>30 \%$ group at baseline compared with the MCF $\leq 30 \%$ group $(58.5 \% \pm 10.8 \%$ vs. $54.2 \% \pm 11.9 \%, \mathrm{P}=0.042)$ but LVEF at LP follow-up was not $(57 \% \pm 8 \%$ vs. $56 \% \pm 10 \%, \mathrm{P}=\mathrm{NS}$, respectively). Overall LVEF slightly increased during follow-up $(56 \% \pm 11 \%$ to
$57 \% \pm 9 \%, \mathrm{P}=\mathrm{NS})$. Nevertheless, after dichotomization according to baseline MCF $(\leq 30 \%$ or $>30 \%)$, the improvement was only a non-significant trend in the MCF $\leq 30 \%$ group $(53 \% \pm 12 \%$ to $56 \% \pm 10 \%, \mathrm{P}=0.07)$. Finally, overall LV RWT decreased over time $(50.3 \% \pm 9.9 \%$ to $48.4 \% \pm 7.5 \%, \mathrm{P}=0.05)$.

\section{Discussion}

To our knowledge, this is the first study assessing MCF for predicting 1-year mortality in a cohort of patients with AS undergoing TAVR.

The most relevant findings of this study are: (I) in patients with severe AS undergoing TAVR, the presence of 
Table 4 Association with all-cause mortality (Cox regression analysis)

\begin{tabular}{|c|c|c|c|}
\hline Variables & $\mathrm{HR}$ & $95 \% \mathrm{Cl}$ & $P$ value \\
\hline \multicolumn{4}{|l|}{ Univariate } \\
\hline Age & 1.03 & $0.95-1.13$ & 0.39 \\
\hline Male sex & 0.89 & $0.37-2.16$ & 0.80 \\
\hline EuroScore II $\geq 5$ & 2.08 & $0.88-4.90$ & 0.09 \\
\hline MCF $\leq 30 \%$ & 3.72 & $1.36-10.17$ & 0.01 \\
\hline$E F \leq 50 \%$ & 1.45 & $0.42-4.95$ & 0.55 \\
\hline $\mathrm{MG}<40 \mathrm{mmHg}$ & 1.20 & $0.48-3.02$ & 0.69 \\
\hline \multicolumn{4}{|l|}{ Multivariate } \\
\hline Age & 1.04 & $0.95-1.14$ & 0.30 \\
\hline EuroScore II $\geq 5$ & 1.97 & $0.80-4.82$ & 0.13 \\
\hline $\mathrm{MCF} \leq 30 \%$ & 2.76 & $1.03-7.77$ & 0.04 \\
\hline LA volume index $\geq 40 \mathrm{~mL} / \mathrm{m}^{2}$ & 3.10 & $0.89-10.77$ & 0.07 \\
\hline
\end{tabular}

$\mathrm{HR}$, hazard ratio; $\mathrm{Cl}$, confidence interval; BMI, body mass index; MCF, myocardial contraction fraction; EF, ejection fraction; MG, mean gradient; LA, left atrial; LV, left ventricular.
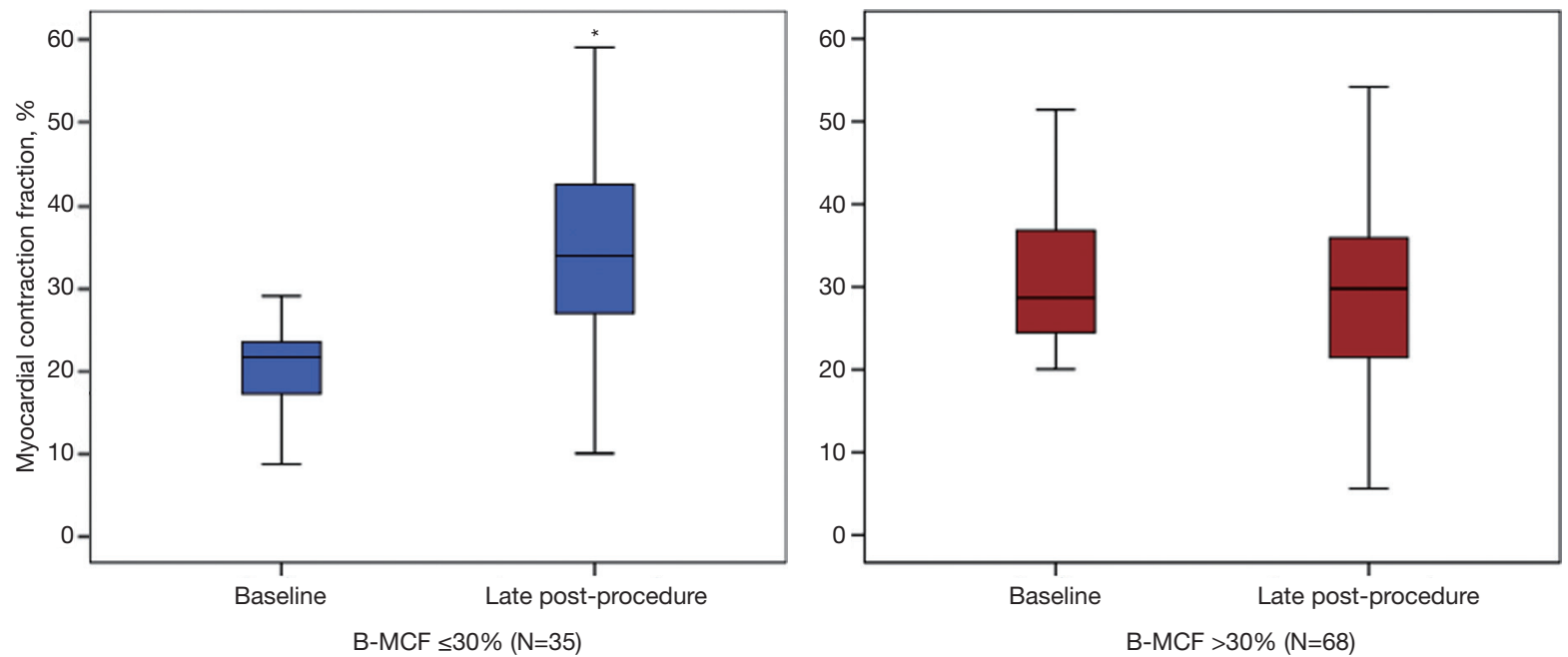

Figure 2 Evolution of MCF according to its baseline value. *, $\mathrm{P}<0.05$ vs. baseline; Whiskers represent maximum and minimum values. MCF, myocardial contraction fraction.

low MCF with mean LVEF $>50 \%$ is prevalent (44\%); (II) patients with high MCF at baseline had better survival than those with low MCF; (III) low MCF at baseline was the only independent predictor of late mortality; and (IV) MCF increased following TAVR only in the group of patients with low MCF at baseline and when TF approach was 

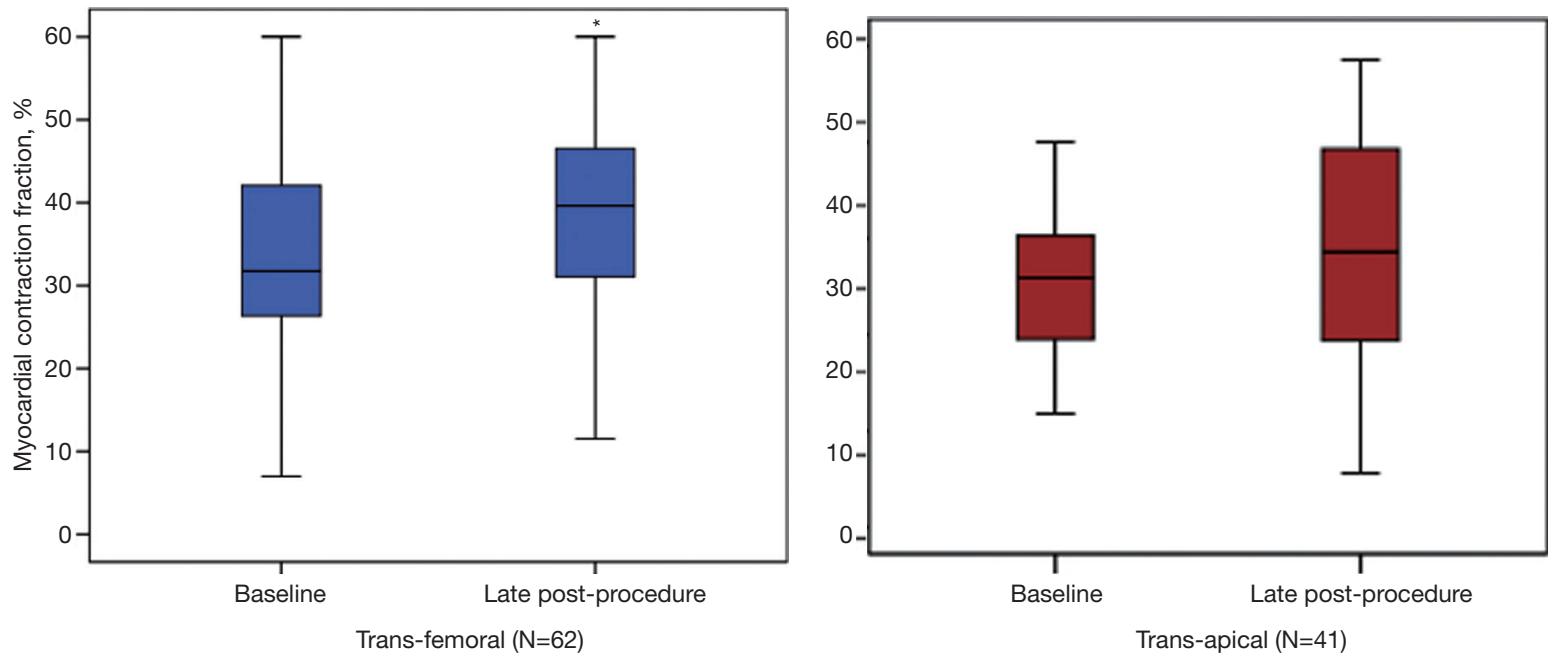

Figure 3 Evolution of myocardial contraction fraction according to the approach: transapical versus transfemoral. *, $\mathrm{P}<0.05$ vs. baseline; Whiskers represent maximum and minimum values.
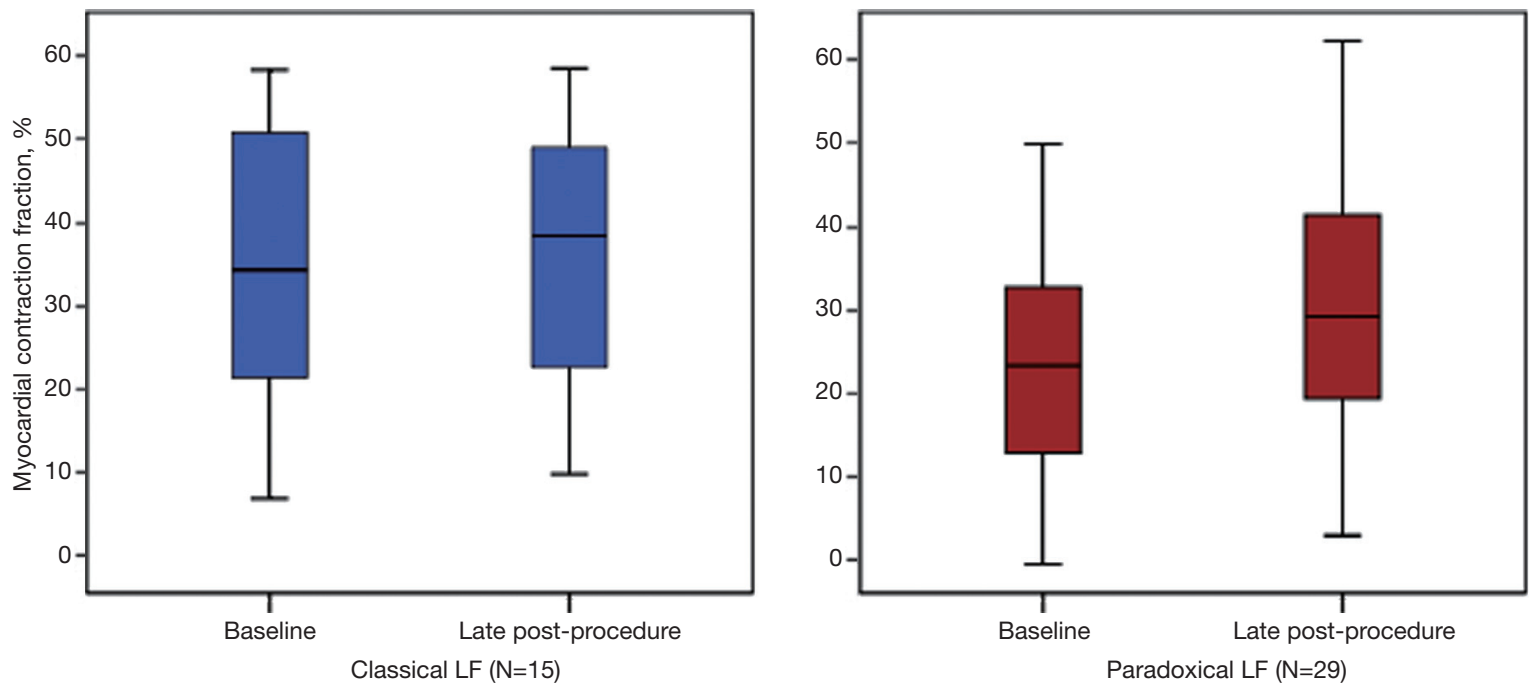

Figure 4 Evolution of myocardial contraction fraction according to the low flow pattern: classical low flow (LF) (LVEF $<50 \%$ and SVi $<35 \mathrm{~mL} / \mathrm{m}^{2}$ ) vs. paradoxical LF (LVEF $\geq 50 \%$ and $\mathrm{SVi}<35 \mathrm{~mL} / \mathrm{m}^{2}$ ). Whiskers represent maximum and minimum values. LVEF, left ventricular ejection fraction; SVi, stroke volume index.

performed.

We had a high mortality rate in this cohort. However, this was mainly driven by perioperative mortality within the first 30 days. Our cohort was a high-risk population with inadequate femoral access in some of the patients and multiple comorbidities requiring transapical access in $40 \%$ of the cases, something very unusual during these days with improvement in ultra-low delivery profile (14 French).

In order to adjust patient selection and therefore, improve short and long-term clinical outcomes following TAVR, reversibility of cardiac function after TAVR has been a main focus of several research groups in the last years. Typically, the removal of afterload by TAVR improves cardiac function (LVEF) and structure by means of a reduction in LV EDV and LV ESV together with reductions in LV mass (17). On the other side, patients who fail to achieve this myocardial structural change are at a greater risk of death during the first-year post TAVR (18). 
Besides cardiac remodeling, LVEF itself may not precisely illustrate the real $\mathrm{LV}$ compromise in the setting of severe AS. Furthermore, LVEF may persist "preserved" for a long period despite reduced myocardial performance owing to preload reserve (19) or changes in LV geometry (20). For example, previous studies have shown that both reduced LVEF and low aortic valve gradient (AVG) at baseline are associated with increased mortality and HF readmissions after TAVR; however, these variables were not fully adjusted for $\mathrm{SV}(21,22)$. Low flow in patients with HF can be secondary to LV pump failure; however, it may also be due to high afterload with restrictive physiology, pronounced concentric hypertrophy, and reduced LV compliance and filling.

MCF equation adjusts SV to LV mass, therefore, diminishing ASE formula bias. As a measure of myocardial shortening, SV is most appropriately assessed relative to the myocardial volume, because it is the myocardium that shortens. MCF is an expression of the magnitude of myocardial contraction during systole relative to total myocardial volume, albeit the myocardium itself has not experienced volume reduction. In fact, it is a measure of cardiac efficiency since it relates cardiac output to myocardial size.

In the present study, low MCF was associated with increased all-cause mortality risk following TAVR compared with higher, i.e., "normal" MCF. Hence, low MCF could reflect a late stage of the disease making interventions futile despite successful procedure. Several clinical findings such as an elevated myocardial fibrosis burden could partially explain these differences between groups (23). Relieving the heart from pressure overload by TAVR is generally beneficial and translates into improved survival lead by both improvement in $\mathrm{SV}$ and/or early regression in $\mathrm{LV}$ mass post-TAVR (24).

We previously mentioned the strong correlation between MCF and strain in different cardiac diseases. Strain imaging with speckle tracking echocardiography has emerged as a useful test to assess LV myocardial deformation and LV contractility especially in patients with excessive LV hypertrophy. GLS can detect latent LV systolic dysfunction which can be useful to identify high-risk cohorts. In fact, it has already shown incremental prognostic value in patients with AS and preserved LVEF (25). After TAVR there is an immediate improvement in GLS (26) especially in patients with higher baseline mean pressure gradient being a surrogate of the contractile reserve (27).

Patients with severe AS and HF are being increasingly managed with TAVR. Cardiac adaptive hypertrophy is a common physiological mechanism where both $\mathrm{LV}$ wall thickness and $L V$ mass tend to increase in order to maintain "normal" wall stress according to Laplace's Law. However, maladaptive hypertrophy and dilatation of the myocardium together with a reduction in SV are common in nearly any advanced cardiac disease and portends dismal prognosis at follow-up.

Differentiating physiological from pathological hypertrophy is critical when evaluating patients with dyspnea and new-onset HF suspicion. For example, in either form of CA, the dominant imaging finding is the appearance of cardiac "hypertrophy". Both AS and CA are diseases from the elderly that usually coexist together. Data from postmortem studies in unselected subjects indicate a prevalence of CA of $22 \%$ to $25 \%$ in subjects older than 80 years of age (28). Moreover, the relationship between AS and transthyretin (ATTR) CA in a cohort of patients undergoing TAVR has recently been described (29). Concomitant ATTR-CA was prevalent (16\%) and was associated with a severe AS low-flow low-gradient variant. Furthermore, these patients presented severe diastolic dysfunction, more severe impairment in GLS and lower MCF. We would like to emphasize the importance of discriminating CA from other forms of LV hypertrophy when evaluating patients before TAVR. Prospective assessment of TAVR outcomes in patients with AS and CA should be performed in larger registries.

We had a low rate of HF readmissions after TAVR, less than reported in the literature (30). We believe other variables such as baseline pulmonary hypertension or requirement for blood transfusions during TAVR may explain these discrepancies (31). Although patients who died were excluded from this end-point analysis, patients with readmissions for $\mathrm{HF}$ are expected to die more at early and late follow-up.

We believe further research involving both MCF and improvements in SV definitions will improve risk stratification in patients with "preserved" LVEF undergoing TAVR.

\section{Clinical implications}

Our study suggests that the measurement of baseline MCF could be a non-invasive tool to assess the immediate hemodynamic benefit of TAVR and so on, predict the risk of perioperative and late mortality after adjusting for several clinical covariables. Although cardiac imaging novel 
techniques such as assessment of GLS by speckle tracking, quantification of myocardial reserve by dobutamine stress echocardiography and the identification of myocardial fibrosis by cardiac magnetic resonance (CMR) may be useful to identify, prior to TAVR, patients who may be at higher risk of persistent low MCF after TAVR; this echocardiographic equation could be easily integrated to risk stratification scores prior to TAVR. As with LVEF, MCF is an index with normal values similar to LVEF, making it simple for clinical use. Noteworthy, our results would suggest that beyond elevated surgical risk scores (i.e., EuroScore II), only patients with high MCF at baseline would be suitable for undergoing TAVR, at least in the high- risk population (EuroScore II $\geq 7$ ) (32).

Finally, we cannot establish whether only low MCF accounts for adverse clinical outcomes or just a surrogate of worse clinical status due to comorbidities such as severe mitral regurgitation, atrial fibrillation, etc. which may preclude irreversible myocardial damage prior to intervention. Hence, further studies are needed to determine whether baseline low MCF should become a central echocardiographic parameter for indexing $\mathrm{LV}$ function.

\section{Strengths and limitations}

The present evaluation was a single-center study, and the analysis and collection of the data were retrospective leading to a few missing samples. Doppler echocardiographic estimation of SVi may be subject to measurement errors, particularly by technical issues such as non-parallel Doppler acquisition angles, sampling of non-laminar flow, and Doppler envelope contamination. Further error may be introduced by mixed regurgitant valve disease, beat-beat variability during arrhythmia, and pressure recovery in small aortic roots. Moreover, AVA calculations are generally based on the continuity equation assuming the LVOT to be spherical when in fact it may be elliptical, becoming more asymmetrical with age (nodular calcium deposition, etc.).

LV mass and volumes were not directly measured, and therefore true volumetric chamber data are lacking. Although the techniques used to estimate $L V$ volumes and mass have been previously validated, they are estimates and not true measures of ventricular volumes and therefore subject to error. However, the main objective of the present study was to demonstrate that MCF, even if measured by means of the simplest method, can predict adverse outcomes.
Finally, we could not account for other variables beyond $\mathrm{MCF}$ at baseline for establishing a cause-effect relationship for chronic heart failure (CHF) readmissions. Probably, clinical and periprocedural variables may impact on this outcome and cannot be systematically excluded.

\section{Conclusions}

In conclusion, $\mathrm{MCF}$, an index of myocardial fiber function, is superior to LVEF in predicting overall survival 1-year after TAVR.

\section{Acknowledgments}

None.

\section{Footnote}

Conflicts of Interest: The authors have no conflicts of interest to declare.

Ethical Statement: This study was approved by the Hospital Italiano Institutional Review Board (IRB No. 00010193), and the requirement for informed consent was waived.

\section{References}

1. Leon MB, Smith CR, Mack M, et al. Transcatheter aorticvalve implantation for aortic stenosis in patients who cannot undergo surgery. N Engl J Med 2010;363:1597-607.

2. Smith CR, Leon MB, Mack MJ, et al. Transcatheter versus surgical aortic-valve replacement in high-risk patients. $\mathrm{N}$ Engl J Med 2011;364:2187-98.

3. Adams DH, Popma JJ, Reardon MJ, et al. Transcatheter aortic-valve replacement with a self-expanding prosthesis. N Engl J Med 2014;370:1790-8.

4. Kodali SK, Williams MR, Smith CR, et al. Two-year outcomes after transcatheter or surgical aortic-valve replacement. N Engl J Med 2012;366:1686-95.

5. Burns RJ, Gibbons RJ, Yi Q, et al. The relationships of left ventricular ejection fraction, end-systolic volume index and infarct size to six-month mortality after hospital discharge following myocardial infarction treated by thrombolysis. J Am Coll Cardiol 2002;39:30-6.

6. Kramer DG, Trikalinos TA, Kent DM, et al. Quantitative evaluation of drug or device effects on ventricular remodeling as predictors of therapeutic effects on mortality in patients with heart failure and reduced 
ejection fraction: A meta-analytic approach. J Am Coll Cardiol 2010;56:392-406.

7. Levy F, Laurent M, Monin JL, et al. Aortic valve replacement for low-flow/low-gradient aortic stenosis operative risk stratification and long-term outcome: a European multicenter study. J Am Coll Cardiol 2008;51:1466-72.

8. Himbert D, Vahanian A. Transcatheter aortic valve replacement for patients with heart failure. Heart Fail Clin 2015;11:231-42.

9. Elmariah S, Palacios IF, McAndrew T, et al. Outcomes of transcatheter and surgical aortic valve replacement in high risk patients with aortic stenosis and left ventricular dysfunction. Results from the Placement of Aortic Transcatheter Valves (PARTNER) trial (cohort A). Circ Cardiovasc Interv 2013;6:604-14.

10. King DL, El-Khoury Coffin L, et al. Myocardial contraction fraction: a volumetric index of myocardial shortening by freehand three-dimensional echocardiography. J Am Coll Cardiol 2002;40:325-9.

11. Milani P, Dispenzieri A, Gertz MA, et al. In patients with light-chain (AL) amyloidosis myocardial contraction fraction (MCF) is a simple, but powerful prognostic measure that can be calculated from a standard echocardiogram (ECHO) (abstr). Blood 2015;126:1774.

12. Tendler A, Helmke S, Teruya S, et al. The myocardial contraction fraction is superior to ejection fraction in predicting survival in patients with AL cardiac amyloidosis. Amyloid 2015;22:61-6.

13. Maurer MS, Koh WJ, Bartz TM, et al. Relation of the Myocardial Contraction Fraction, as Calculated from M-Mode Echocardiography, With Incident Heart Failure, Atherosclerotic Cardiovascular Disease and Mortality (Results from the Cardiovascular Health Study). Am J Cardiol 2017;119:923-8.

14. Arenja N, Riffel JH, Fritz T et al. Diagnostic and Prognostic Value of Long-Axis Strain and Myocardial Contraction Fraction Using Standard Cardiovascular MR Imaging in Patients with Nonischemic Dilated Cardiomyopathies. Radiology 2017;283:681-91.

15. Baumgartner H, Hung J, Bermejo J, et al.

Recommendations on the echocardiographic assessment of aortic valve stenosis: A focused update from the European Association of Cardiovascular Imaging and the American Society of Echocardiography. Eur Heart J Cardiovasc Imaging 2017;18:254-75.

16. Lang RM, Badano LP, Mor-Avi V, et al. Recommendations for cardiac chamber quantification by echocardiography in adults: an update from the American Society of Echocardiography and the European Association of Cardiovascular Imaging. Eur Heart J Cardiovasc Imaging 2015;16:233-70. Erratum in: Recommendations for Cardiac Chamber Quantification by Echocardiography in Adults: An Update from the American Society of Echocardiography and the European Association of, Cardiovascular Imaging. [Eur Heart J Cardiovasc Imaging 2016] Corrigendum. [Eur Heart J Cardiovasc Imaging 2016].

17. La Manna A, Sanfilippo A, Capodanno D, et al. Left ventricular reverse remodeling after transcatheter aortic valve implantation: a cardiovascular magnetic resonance study. J Cardiovasc Magn Reson 2013;15:39.

18. Sato K, Kumar A, Jones BM, et al. Reversibility of Cardiac Function Predicts Outcome After Transcatheter Aortic Valve Replacement in Patients With Severe Aortic Stenosis. J Am Heart Assoc 2017. doi: 10.1161/ JAHA.117.005798.

19. Krayenbuehl HP, Hess OM, Ritter M, et al. Left ventricular systolic function in aortic stenosis. Eur Heart J 1988;9:19-23.

20. Delgado V, Tops LF, van Bommel RJ, et al. Strain analysis in patients with severe aortic stenosis and preserved left ventricular ejection fraction undergoing surgical valve replacement. Eur Heart J 2009;30:3037-47.

21. Baron SJ, Arnold SV, Herrmann HC, et al. Impact of Ejection Fraction and Aortic Valve Gradient on Outcomes of Transcatheter Aortic Valve Replacement. J Am Coll Cardiol 2016;67:2349-58.

22. Conrotto F, D'Ascenzo F, D'Amico M, et al. Outcomes of patients with low-pressure aortic gradient undergoing transcatheter aortic valve implantation: A Meta-analysis. Catheter Cardiovasc Interv 2017;89:1100-6.

23. Weidemann F, Herrmann S, Störk S, et al. Impact of myocardial fibrosis in patients with symptomatic severe aortic stenosis. Circulation 2009;120:577-84.

24. Lindman BR, Stewart WJ, Pibarot P, et al. Early regression of severe left ventricular hypertrophy after transcatheter aortic valve replacement is associated with decreased hospitalizations. JACC Cardiovasc Interv 2014;7:662-73.

25. Kusunose K, Goodman A, Parikh R, et al. Incremental prognostic value of left ventricular global longitudinal strain in patients with aortic stenosis and preserved ejection fraction. Circ Cardiovasc Imaging 2014;7:938-45.

26. Lozano Granero VC, Fernandez Santos S, FernandezGolfín C, et al. Immediate improvement of left ventricular mechanics following transcatheter aortic valve replacement. Cardiol J 2018;25:487-94. 
27. Corrigan FE, Zhou X, Lisko JC, et al. Mean Aortic pressure gradient and global longitudinal strain recovery after transcatheter aortic valve replacement - A retrospective analysis. Hellenic J Cardiol 2018;59:268-71.

28. Ueda M, Horibata Y, Shono M, et al. Clinicopathological features of senile systemic amyloidosis: an ante- and postmortem study. Mod Pathol 2011;24:1533-44.

29. Castaño A, Narotsky DL, Hamid N, et al. Unveiling transthyretin cardiac amyloidosis and its predictors among elderly patients with severe aortic stenosis undergoing transcatheter aortic valve replacement. Eur Heart J 2017;38:2879-87.

30. Nombela-Franco L, del Trigo M, Morrison-Polo G, et al.

Cite this article as: Romeo FJ, Seropian IM, Arora S, Vavalle JP, Falconi M, Oberti P, Kotowicz V, Agatiello CR, Berrocal DH. Prognostic impact of myocardial contraction fraction in patients undergoing transcatheter aortic valve replacement for aortic stenosis. Cardiovasc Diagn Ther 2020;10(1):12-23. doi: 10.21037/cdt.2019.05.02
Incidence, causes, and predictors of early ( $\leq 30$ days) and late unplanned hospital readmissions after transcatheter aortic valve replacement. JACC Cardiovasc Interv 2015;8:1748-57.

31. Durand E, Doutriaux M, Bettinger N, et al. Incidence, Prognostic Impact, and Predictive Factors of Readmission for Heart Failure After Transcatheter Aortic Valve Replacement. JACC Cardiovasc Interv 2017;10:2426-36.

32. Arangalage D, Cimadevilla C, Alkhoder S, et al. Agreement between the new EuroSCORE II, the Logistic EuroSCORE and the Society of Thoracic Surgeons score: implications for transcatheter aortic valve implantation. Arch Cardiovasc Dis 2014;107:353-60. 